\title{
Competencias Clave del Profesorado: diseño y validación del marco global competencial de los docentes
}

\author{
Laia Lluch Molins ${ }^{1}$ (D), Elena Cano García ${ }^{2}$ (D) Laura Pacheco Villarroel ${ }^{2}$ (D)
}

Artículo de estudios y tendencias. Recibido: 06/04/2020. Aceptado:12/05/2020. Publicación avanzada: 26/06/2020. Publicado: 01/07/2020

\section{Resumen}

INTRODUCCIÓN. A partir del Consejo Europeo de Lisboa (2000) se han realizado grandes esfuerzos en las instituciones de educación superior para implementar el enfoque basado en competencias. Por ejemplo, han sido creados marcos de competencias clave del profesorado; a pesar de que varían mucho no solo entre países, sino también entre instituciones dentro de un mismo país. De ahí la necesidad de un modelo más global de marco competencial para docentes, alineando, así, la pre-service teacher education con la in-service teacher education o formación permanente y facilitando de este modo el desarrollo profesional a lo largo de la vida (educación para toda la vida).

MÉTODO. En esta contribución, se presenta el proceso seguido para diseñar y validar el marco teórico global de las competencias clave del profesorado.

RESULTADOS. Se detallan los pasos seguidos para la construcción de este marco de referencia, empezando por una revisión sistemática de marcos competenciales existentes en distintas zonas del mundo.

DISCUSIÓN. Se pretende asegurar así la transferibilidad de este marco a otros contextos y la armonización global; así como se especifican los diferentes procesos de validación para fomentar su uso en futuros estudios e investigaciones, promoviendo así la armonización global.

\section{Palabras clave}

Competencias clave del profesorado; Desarrollo profesional; Educación superior; Educación para toda la vida; Formación.

\section{Datos de los autores}

${ }^{1}$ Universitat de Barcelona, España. Contacto para la correspondencia: laia.Iluch@ub.edu

2 Universitat de Barcelona, España.

Referencia recomendada

Lluch, L., Cano, E., y Pacheco, L. (2020). Competencias Clave del Profesorado: diseño y validación del marco global competencial de los docentes. REIRE Revista d'Innovació i Recerca en Educació, 13(2), 1-16. https://doi.org/10.1344/reire2020.13.231230

(C) 2020 Laia Lluch, Elena Cano y Laura Pacheco. Este artículo es de acceso abierto sujeto a la licencia Reconocimiento 4.0 Internacional de Creative Commons, la cual permite utilizar, distribuir y reproducir por cualquier medio sin restricciones siempre que se cite adecuadamente la obra original. Para ver una copia de esta licencia, visite http://creativecommons.org/licenses/by/4.0/ 
L. Lluch, E. Cano y L. Pacheco. Competencias Clave del Profesorado: diseño y validación del marco global competencial de los docentes

Títol

Competències clau del professorat: disseny i validació del marc global competencial dels docents

Resum

INTRODUCCIÓ. Des del Consell Europeu de Lisboa (2000) s'han fet grans esforços a les institucions d'educació superior per implementar l'enfocament basat en competències. S'han creat, per exemple, marcs de competències clau del professorat, tot i que varien molt no només entre països, sinó també entre institucions dins d'un mateix país. D'aquí sorgeix la necessitat d'un model més global de marc competencial per a docents per alinear tota la carrera docent, és a dir, la pre-service teacher education amb la inservice teacher education o formació permanent, i facilitar d'aquesta manera el desenvolupament professional al llarg de tota la vida (educació per a tota la vida).

MÈTODE. En aquesta comunicació es presenta el procés seguit per dissenyar i validar el marc teòric global de les competències clau del professorat.

RESULTATS. S'hi detallen els passos seguits per a la construcció d'aquest marc de referència, començant per una revisió sistemàtica de marcs competencials existents en diferents zones del món.

DISCUSSIÓ. Es pretén assegurar, així, la transferibilitat d'aquest marc a altres contextos i l'harmonització global; s'hi especifiquen, per exemple, els diferents processos de validació per fomentar-ne l'ús en futures recerques i investigacions, i promoure aquesta harmonització.

Paraules clau

Competències clau del professorat; Desenvolupament professional; Educació superior; Educació per a tota la vida; Formació.

\section{Title}

Teachers' Key Competences: design and validation of the global teachers' competence-based framework

Abstract

INTRODUCTION. Since the European Council meeting in Lisbon in 2000, higher education institutions have made considerable efforts to implement a competence-based approach. For example, teachers' key competence frameworks have been created, even though they vary greatly not only between countries, but also between institutions within the same country. These differences highlight the need for a more global model of competence framework for teachers, aligning pre-service teacher education with in-service teacher education or permanent training and thus encouraging professional lifelong learning.

METHOD. This contribution presents the process followed to design and validate the global theoretical framework of teachers' key competences, designed in a European Project co-funded by the European Commission.

RESULTS. The steps followed for the construction of this reference framework are detailed. The paper starts with a systematic review of existing competence frameworks in different geographical areas.

DISCUSSION. The aim is to ensure the transferability of this framework to other contexts and to promote global harmonization. The different validation processes are specified in order to encourage their use in future studies and research.

Keywords

Higher education; Lifelong learning; Professional development; Teachers' key competences; Training. 


\section{Introducción}

En el campo de la formación docente, algunos trabajos han tratado de identificar las competencias clave de los docentes y se han desarrollado varios marcos competenciales tratando de identificar los perfiles competenciales docentes. Si bien algunos de estos marcos son específicos de cada país, otros tienen un alcance más amplio (Caena, 2011) o varían en cuanto a sus resultados o niveles de implementación (Olsen, 2005; Stryven y De Meyst, 2010). Por ello se hace necesario un marco global integrado.

Por otra parte, no todos los análisis realizados hasta el momento establecen niveles de desempeño a lo largo de la carrera docente. Con este fin, el Council of the European Union (2014) recomienda que los organismos competentes definan marcos referenciales de competencias y cualidades profesionales requeridas para el profesorado en cada fase de su carrera docente. En este sentido, se han emprendido algunas acciones para presentar las competencias clave de los docentes en base a rúbricas que establezcan estadios o fases de desarrollo. Este es el caso, a modo de ilustración, del Marco común de competencias profesionales docentes coordinado por el Centro Autonómico de Formación e Innovación (CAFI) (http://www.edu.xunta.gal/centros/erasmusplusmccpd/en/node/48).

Independientemente de la diversidad de marcos, existe un acuerdo general de que las competencias de los profesores deben desarrollarse a lo largo de toda la carrera docente (Bokdam et al., 2014; Caena, 2011; 2014), también para el desarrollo futuro de los centros educativos y para la mejora del sistema educativo. Es decir, debe haber una alineación entre la formación inicial (pre-service teacher education) y la in-service teacher education, de modo que se favorezca el desarrollo profesional, así como el aprendizaje continuo a lo largo de la vida, para perseguir con la formación una mejora de la calidad educativa en los centros educativos (Hanushek, 2011).

En consecuencia, la formación inicial del profesorado tiene que desarrollar el nivel de competencia necesario en cada competencia clave para garantizar que los profesores noveles y experimentados tengan las habilidades suficientes para desempeñar su profesión de manera eficiente (Conner y Sliwka, 2014; Darling-Hammond y Bransford, 2005). El Council of the European Union (2014) indica que la formación inicial debería proporcionar a los docentes las competencias clave que les permitan ser excelentes en su práctica, al tiempo que los motive a reforzarlas y actualizarlas a lo largo de su carrera profesional. De ahí que la formación inicial y el desarrollo continuo tienen que establecer vínculos para asegurar que los maestros continúen desarrollando sus competencias a lo largo de su carrera profesional.

En el caso de la formación inicial del profesorado en España, "los cambios que se están produciendo en la sociedad inciden en la demanda de una redefinición del trabajo del profesor y seguramente de la profesión docente, de su formación y de su desarrollo profesional" (Marcelo, 2001, p. 551). En el marco del aprendizaje a lo largo de toda la vida, no solo es importante identificar las competencias clave y la capacitación de los docentes para desarrollarlas, sino también que las instituciones encargadas de la formación se responsabilicen de su desarrollo competencial, certificación y validación a través de actividades de formación. Para que docentes de las facultades de Educación se responsabilicen, deben conocer y participar en el establecimiento de las competencias que deben desarrollar en los futuros maestros, así como ayudar a los estudiantes a detectar el nivel de competencia actual en el cual se encuentra cada uno. 
Sin embargo, las competencias clave de los docentes y sus implicaciones no siempre se presentan con claridad, probablemente, porque no existe una sola comprensión de la educación y la enseñanza (Black y Wiliam, 1998; Olsen, 2005). En esta línea, se detecta la necesidad de una estructura que encamine la oferta hacia un modelo más global, que dé cobertura a todas las necesidades formativas del profesorado y, al mismo tiempo, responda a las recomendaciones de la Unión Europea en materia competencial (Council of the European Union, 2014). Igualmente animan a la creación de marcos competenciales para formadores de docentes, que sirvan de punto de partida para la formación inicial, la formación continua y los procesos de enseñanza y aprendizaje en todo el mundo refuerza la transferencia entre países y contribuye a compensar la diversidad fruto de las diferencias en los sistemas educativos, diferentes regiones y países, asegurando así la equidad educativa. De ahí la necesidad de un modelo más global de marco competencial para docentes, que facilite la transparencia y la movilidad, que impulse políticas de formación y medidas transnacionales armónicas y bien alineadas con los actuales retos educativos. Independientemente de la diversidad de marcos diseñados, hay un consenso general en cuanto a que las competencias clave del profesorado deben desarrollarse a lo largo de toda la carrera docente.

El objetivo de esta contribución es presentar el proceso seguido para diseñar y validar el marco teórico global de las competencias clave del profesorado, diseñado en el Proyecto Europeo "Building up Chinese Primary Teachers' Key Competences through a Global Competence-based Framework" con referencia 586415-EPP-1-2017-1-ES-EPPKA2-CBHE-JP y cofinanciado por la Unión Europea. La necesidad de desarrollar un proyecto que cree un Marco Global de las Competencias Clave del profesorado en China es mayor que en otros contextos, pues el sistema educativo del país se caracteriza por la especialización de los maestros en áreas de conocimiento específicas, es decir, por una formación del profesorado más tradicional y, consecuentemente, una enseñanza en las aulas de primaria donde predomina la transmisión de conocimientos. Esta enseñanza se estructura por asignaturas o áreas de conocimiento, por lo que el conocimiento del contenido de una especialidad adopta más relevancia que el hecho de ayudar a los alumnos a desarrollar las competencias básicas para vivir y adaptarse a los constantes cambios económicos, ambientales y sociales del siglo xxi (OECD, 2018).

En esta línea, la formación del profesorado para los maestros en China se focaliza en aquella disciplina en la cual se quieran especializar (Ding, 2016). De ahí que en las Facultades de Educación hay varias especializaciones, tales como lengua y literatura, historia, inglés o matemáticas, entre otras. Aunque cada facultad desarrolla su propio currículum de formación del profesorado, pues no hay un módulo unificado de formación de maestros de primaria elaborado por el Ministerio Central de Educación de China, generalmente estos consisten en un año de conocimientos generales y tres años de especialización en una disciplina (Yang, 2009). Los estudiantes que deseen obtener el certificado de cualificación docente tienen que presentarse al examen nacional de cualificación docente, y para ello deben escoger una de las posibles especializaciones (Ding, 2016).

Varios investigadores chinos afirman que la formación del profesorado está demasiado centrada en el conocimiento de la materia, con lo cual carece de formación para el correcto desarrollo personal del alumno de primaria (Huang, 2009; Yang, 2009; Zhang, 2013; Zhou, Liji, Deng y Xue, 2007, citados en Ding, 2016). Tal y como afirma la literatura, "no es posible un cambio metodológico sin una adecuada formación del profesorado" (Calvo-Bernardino y Mingorance-Arnáiz, 2009, p. 334). Por consiguiente, un cambio en la formación del profesorado en un contexto como China es esencial para conseguir la formación integral del alumnado que le permitirá, no solo conocer el contenido de las materias, sino también aplicarlo en varios 
contextos reales. A pesar de que en los últimos años se han llevado a cabo varios proyectos desde el gobierno -como el programa "Excellent teachers" (Ministerio de Educación de China, 2014, citado en Ding, 2016) - o el National Teacher Training Programme for Teachers in Kindergarten, Primary and Secondary Schools -impulsado desde 2010 por el Ministerio de Educación de China - y desde universidades del país para desarrollar una formación más transversal de los maestros de educación primaria, China carece de experiencia consolidada para guiar la creación de un currículum competencial para la formación del profesorado.

Por lo tanto, con el fin de cambiar la formación del profesorado en China hacia una formación más transversal, que ayude a los alumnos de primaria a desarrollar las competencias clave del siglo xxi establecidas por DeSeCo (OECD, 2005), se consideró necesaria la colaboración de varios países de la Unión Europea con China para crear un Marco Global de las Competencias Clave de los Docentes a partir del cual se desarrollase un currículum basado en las competencias para la formación del profesorado.

No obstante, el marco se crea en el entorno del proyecto “Building up Chinese Primary Teachers' Key Competences through a Global Competence-based Framework", este pretende ser, como bien dice el nombre, un marco global para todos los docentes independientemente del país en el cual desempeñen su labor. Es por esto que este documento de referencia se desarrolla a partir del análisis de otros marcos pertenecientes a países de distintas zonas del mundo para asegurar así la transferibilidad de este a otros contextos. Por lo tanto, este marco global es una contribución necesaria en el contexto actual para promover la armonización global y la comparabilidad de las calificaciones, así como la existencia de continuidad entre las diferentes etapas del aprendizaje a lo largo de la vida -carrera inicial, temprana y desarrollo-; lo cual es válido para cualquier país, pero especialmente relevante para China.

\section{Metodología}

La presente contribución presenta el diseño y la validación del Marco Global de las Competencias Clave de los Docentes diseñado a partir del proyecto europeo “Building up Chinese Primary Teachers' Key Competences through a Global Competence-based Framework". La creación y validación de este marco teórico global sigue los siguientes pasos (véase cuadro 1):

\section{Cuadro 1}

Pasos para la construcción del marco. Elaboración propia.

1. Selección de documentos

2. Análisis sistemático de las propuestas de competencias

3. Decisiones respecto al modo de formular las competencias

4. Primera versión del marco competencial

5. Validación por parte de expertos

6 . Segunda versión del marco competencial

7. Validación por parte del equipo internacional de socios

8. Tercera versión del marco competencial 
L. Lluch, E. Cano y L. Pacheco. Competencias Clave del Profesorado: diseño y validación del marco global competencial de los docentes

\section{Resultados}

\subsection{Selección de documentos}

Para el diseño del marco competencial se procedió en primer lugar a una revisión sistemática de marcos competenciales. La selección se realizó atendiendo a los criterios siguientes:

a) Ser marcos referentes a las competencias clave del profesorado.

Criterio de exclusión: Marcos referentes a una competencia en particular $-p$. e. competencia digital一.

b) Ser marcos de organizaciones transnacionales o nacionales representativas del colectivo docente o con una clara dimensión institucional y/o de la administración educativa.

Criterio de exclusión: Marcos realizados por investigadores particulares.

c) Ser marcos pertenecientes a diversas áreas geográficas:

Criterio de exclusión: Marcos relativos a países o naciones no pertenecientes al proyecto.

Al tratarse de un proyecto europeo se partió de los marcos de los países pertenecientes al consorcio. Por este motivo, se seleccionaron los marcos competenciales de:

- España -en este caso se aportaron varios marcos, tanto el derivado de la orden ECl/3857/2007, de 27 de diciembre, por la que se establecen los requisitos para la verificación de los títulos universitarios oficiales que habiliten para el ejercicio de la profesión de maestro en educación primaria como el de los grados de la Universidad de Barcelona o como el derivado de un proyecto similar al nuestro, el Proyecto Erasmus de Galicia (http://www.edu.xunta.gal/centros/erasmusplusmccpd/).

- Portugal

- $\quad$ Reino Unido

- Al no existir un marco competencial exclusivo de China, se seleccionaron dos marcos representativos: el del Sudeste asiático y el del GESCl y se añadieron también los marcos nacionales de Taiwán y Hong Kong, con el fin de aumentar la representación de este continente, atendiendo a la naturaleza y propósito del proyecto europeo.

- A la vez, al tratarse de un marco global, se buscó la representación de todos los continentes, para poder reflejar, si procede, las diferencias en la concepción y selección de competencias, motivo por el cual se incluyeron los marcos de los países del $\mathrm{GESCl}^{1}$-para incorporar no solo Corea y China, sino también, en especial, África-, de Australia -en representación de

\footnotetext{
${ }^{1}$ Global e-Schools and Communities Initiative (GESCI) es una organización internacional sin ánimo de lucro fundada bajo la recomendación del grupo de trabajo de las Naciones Unidas sobre las Tecnologías de la Información y la Comunicación (TIC). GESCI se estableció en la primera cumbre mundial sobre la sociedad de la Información en 2003. Su misión es trabajar con los gobiernos de los países que la forman para desarrollar e implementar modelos de buenas prácticas basadas en las TIC para una educación de calidad (Park y Tan, 2016).
} 
L. Lluch, E. Cano y L. Pacheco. Competencias Clave del Profesorado: diseño y validación del marco global competencial de los docentes

Oceanía - y de USA - en representación de América del Norte- y los marcos transnacionales de UNESCO, OCDE y Comisión Europea.

Con relación a este criterio, se intentó evitar la sobrerrepresentación de marcos europeos que, por otra parte, eran los más numerosos-, lo cual podría dar lugar a una visión eurocéntrica $y$, por lo tanto, sesgada, de las principales competencias docentes.

\subsection{Análisis sistemático de las propuestas de competencias}

Tras una lectura atenta de los marcos y de sus propuestas de clasificación de competencias, se procedió a un análisis inductivo (véase tabla 1).

Tabla 1

Análisis inductivo de las propuestas de clasificación de las competencias. Elaboración propia.

\begin{tabular}{|c|c|c|}
\hline COMPETENCE LIST & UNESCO & European Commission \\
\hline Subject matter & $\begin{array}{l}\text { 2.1. Technology literacy approach/stage: teachers must } \\
\text { have an excellent knowledge of the curriculum standards } \\
\text { for their subject. } \\
\text { 2.2. Knowledge Deepening approach/stage: teachers } \\
\text { must have a deep knowledge of their subject. }\end{array}$ & Subject matter and curricula. \\
\hline $\begin{array}{l}\text { Pedagogical content } \\
\text { knowledge }\end{array}$ & $\begin{array}{l}\text { 2.2. Knowledge Deepening approach/stage: teachers } \\
\text { must have the ability to apply their knowledge flexibly in } \\
\text { a variety of situations. They must also be able to create } \\
\text { complex problems whose solutions measure students' } \\
\text { understanding. } \\
\text { 2.3. Knowledge Creation approach/stage: teachers must } \\
\text { know about complex human development, such as } \\
\text { cognitive, emotional and physical development. They } \\
\text { must know how, and under which conditions, students } \\
\text { learn best. Teachers must have the skills required to } \\
\text { support these complex processes. } \\
\text { 3.2. Knowledge Deepening approach/stage: teaching is } \\
\text { student-centred in this approach and the teacher's role is } \\
\text { to provide direct instruction in consciously skilled ways } \\
\text { and to structure problem tasks, guide student } \\
\text { understanding, and support student collaborative } \\
\text { projects. In this role teachers must have the skills to help } \\
\text { students create, implement, and monitor project plans } \\
\text { and solutions } \\
\text { 3.3. Knowledge Creation approach/stage: the role of } \\
\text { teachers in this approach is to explicitly model the } \\
\text { learning processes and create situations in which } \\
\text { students apply their developmental skills. }\end{array}$ & $\begin{array}{l}\text { Learning, context, psychology, } \\
\text { education sciences, Pedagogical } \\
\text { Content Knowledge, critical attitude } \\
\text { to practice, epistemological } \\
\text { awareness, metacognitive, adaptive, } \\
\text { process teaching evidence and } \\
\text { monitor. }\end{array}$ \\
\hline
\end{tabular}

(Continúa en la siguiente página.) 
L. Lluch, E. Cano y L. Pacheco. Competencias Clave del Profesorado: diseño y validación del marco global competencial de los docentes

\begin{tabular}{|c|c|c|}
\hline COMPETENCE LIST & UNESCO & European Commission \\
\hline $\begin{array}{l}\text { Information and } \\
\text { Communication } \\
\text { Technologies }\end{array}$ & $\begin{array}{l}\text { 2.1. Technology literacy approach/stage: teachers must } \\
\text { be able to integrate the use of technology into the } \\
\text { curriculum. } \\
\text { 3.1. Technology literacy approach/stage: teachers must } \\
\text { know where, with whom, when (as well as when not) and } \\
\text { how to use ICT for classroom activities and presentations. } \\
\text { 4.1. Technology literacy approach/stage: teachers must } \\
\text { know basic hardware and software operations, as well as } \\
\text { productivity applications software, a web browser, } \\
\text { communications software, presentation software, and } \\
\text { management applications. } \\
\text { 4.2. Knowledge Deepening approach/stage: teachers } \\
\text { must be knowledgeable about a variety of subject- } \\
\text { specific tools and applications and be able to flexibly use } \\
\text { these in a variety of problem-based and project-based } \\
\text { situations. Teachers should be able to use network } \\
\text { resources to help students collaborate, access } \\
\text { information and communicate with external experts in } \\
\text { order to analyze and solve their selected problems. } \\
\text { Teachers should also be able to use ICT to create and } \\
\text { monitor individual and group student project plans. } \\
\text { 4.3. Knowledge Creation approach/stage: teachers must } \\
\text { be able to design ICT-based knowledge communities and } \\
\text { use ICT to support the development of students' } \\
\text { knowledge creation skills and their continuous, reflective } \\
\text { learning. } \\
\text { 5.1.Technology literacy approach/stage: teachers must } \\
\text { be able to use technology with the whole class, small } \\
\text { groups, and individual activities and ensure equitable } \\
\text { access is provided to all students. }\end{array}$ & $\begin{array}{l}\text { Information and Communication } \\
\text { Technologies, use materials. }\end{array}$ \\
\hline $\begin{array}{l}\text { Professional } \\
\text { development }\end{array}$ & $\begin{array}{l}\text { 4. Lifelong learning. } \\
\text { 4.1. Attitudes towards the acquisition of new knowledge } \\
\text { and of learning regarding new technologies. } \\
\text { 4.2. Attitudes towards lifelong learning ang the use of ICT } \\
\text { to improve personal and professional development. } \\
\text { 4.2. Attitudes towards lifelong learning ang the use of ICT } \\
\text { to improve personal and professional development. } \\
\text { 9. Research and development. } \\
\text { 9.1. The ability to do research on pedagogy-ICT. } \\
\text { 9.2. The ability to do research on applying ICT in the } \\
\text { teaching of a specific topic. } \\
\text { 9.3. The ability to develop the ICT competence through } \\
\text { "learning by doing". }\end{array}$ & Ongoing learning and research. \\
\hline Assessment & $\begin{array}{l}\text { 2.1. Technology literacy approach/stage: teachers must } \\
\text { have an excellent knowledge of standard assessment } \\
\text { strategies. } \\
\text { 3.2. Knowledge Deepening approach/stage: teachers } \\
\text { need to use assessment for learning as a basic principle } \\
\text { guiding their practice. }\end{array}$ & Assessment and assess teaching. \\
\hline $\begin{array}{l}\text { Teaching practice } \\
\text { (innovative, varied } \\
\text { and dynamic tasks) }\end{array}$ & & Group dynamics and negotiation. \\
\hline $\begin{array}{l}\text { Classroom } \\
\text { management }\end{array}$ & $\begin{array}{l}\text { 5.2. Knowledge Deepening approach/stage: teachers } \\
\text { must be able to create flexible classroom learning } \\
\text { environments. Within these environments, teachers } \\
\text { must be able to integrate student-centred activities and } \\
\text { flexibly apply technology to support collaboration. }\end{array}$ & $\begin{array}{l}\text { Organization aspects, self-efficacy, } \\
\text { class management, plan and manage } \\
\text { teaching. }\end{array}$ \\
\hline
\end{tabular}

(Continúa en la siguiente página.) 
L. Lluch, E. Cano y L. Pacheco. Competencias Clave del Profesorado: diseño y validación del marco global competencial de los docentes

\begin{tabular}{|c|c|c|}
\hline COMPETENCE LIST & UNESCO & European Commission \\
\hline \multicolumn{3}{|l|}{$\begin{array}{c}\text { Communication } \\
\text { skills }\end{array}$} \\
\hline Inclusive attitude & $\begin{array}{l}\text { 2.3. Knowledge Creation approach/stage: teachers must } \\
\text { anticipate and be able to effectively respond to the } \\
\text { difficulties students encounter. }\end{array}$ & Inclusion and diversity. \\
\hline $\begin{array}{l}\text { Cooperation and } \\
\text { collaboration with } \\
\text { families, } \\
\text { professionals, the } \\
\text { school and the } \\
\text { community }\end{array}$ & $\begin{array}{l}\text { 5.3. Knowledge Creation approach/stage: teachers } \\
\text { should be able to play a leadership role in training and } \\
\text { providing follow-up support to colleagues and in creating } \\
\text { and implementing a vision of their school as a community } \\
\text { based on innovation and continuous learning enriched by } \\
\text { ICT. } \\
\text { 6.2. Knowledge Deepening approach/stage: teachers } \\
\text { must have the skills and knowledge to create and } \\
\text { manage complex projects, collaborate with other } \\
\text { teachers, and make use of networks to access } \\
\text { information, colleagues and outside experts in } \\
\text { supporting their own professional learning. } \\
\text { 6.3. Knowledge Creation approach/stage: teachers, too, } \\
\text { must have the ability, motivation, inclination, } \\
\text { encouragement and support to experiment, continuously } \\
\text { learn and use ICT to build professional learning } \\
\text { communities working toward creating knowledge. }\end{array}$ & Cooperation and collaboration. \\
\hline $\begin{array}{l}\text { Awareness of } \\
\text { policies and laws } \\
\text { that regulate the } \\
\text { school and } \\
\text { education }\end{array}$ & $\begin{array}{l}\text { 1.1. Technology Literacy approach/stage: teachers must } \\
\text { be aware of policies and be able to articulate in } \\
\text { consciously skilled ways how their classroom practices } \\
\text { correspond to and support policy. } \\
\text { 1.2. Knowledge Deepening approach/stage: teachers } \\
\text { must have an in-depth knowledge of national policies } \\
\text { and social priorities, and be able to design, modify, and } \\
\text { implement classroom practices that support these } \\
\text { policies. } \\
\text { 1.3. Knowledge Creation approach/stage: teachers must } \\
\text { understand the intentions of national policies and be } \\
\text { able to contribute to the discussion of education reform } \\
\text { policies and participate in the design, implementation, } \\
\text { and revision of programmes intended to implement } \\
\text { these policies. }\end{array}$ & Institution. \\
\hline Teacher ethics & $\begin{array}{l}\text { 6.3. Knowledge Creation approach/stage: teachers, too, } \\
\text { must have the ability, motivation, inclination, } \\
\text { encouragement and support to experiment, continuously } \\
\text { learn and use ICT to build professional learning } \\
\text { communities working toward creating knowledge. }\end{array}$ & Commitment to pupils' learning. \\
\hline
\end{tabular}

Este primer marco constaba de 12 competencias agrupadas en 3 bloques. Tras el análisis pormenorizado de la primera clasificación y, atendiendo al contenido de las competencias halladas, se decidió prescindir de "Subject matter" para formar parte de uno de los descriptores de la dimensión de planificación, "Pedagogical content knowledge" para ir a los descriptores de planificación y gestión del aula, "Teaching practice" para ir a los descriptores de planificación, y "Awareness of policies and laws that regulate the school and education" para pasar a incluirse como un descriptor de compromiso ético. Además, se comprobó, atendiendo a la frecuencia, que muchos de ellos estructuraban su contenido en cuatro áreas - materias- y que proponían listados de competencias que se fueron anotando de forma sistemática para llegar, finalmente a categorizarse en torno a 9 grandes grupos. 
L. Lluch, E. Cano y L. Pacheco. Competencias Clave del Profesorado: diseño y validación del marco global competencial de los docentes

Materia

- Contenido disciplinar

- Contenido pedagógico

- Conocimiento Tecnologías de la información y la comunicación

- Desarrollo profesional

\section{Competencias}

- Evaluación

- Práctica docente (tareas innovadoras, variadas y dinámicas)

- Gestión de la clase

- Habilidades de comunicación

- TICs

- Actitud inclusiva

- Cooperación y colaboración con las familias, profesionales, la escuela y la comunidad

- Conciencia de las políticas y leyes que regulan la escuela y educación

- Ética del profesor

Esta propuesta de competencias clave emergente se basa en las siguientes premisas:

$\checkmark$ Se pretende tener un marco teórico general de las competencias de los docentes de Educación Primaria.

$\checkmark$ El número de competencias debe reducirse, ya que está destinado a definir las competencias clave.

$\checkmark$ Se debe hacer un esfuerzo en la redacción de cada competencia, para que sean lo más comprensibles posible.

\subsection{Decisiones respecto al modo de formular las competencias}

Aunque en un primer momento, estas categorías emergentes tenían nombres largos, compuestos de las diversas denominaciones o similares etiquetas que cada uno de los marcos analizados había propuesto, finalmente se tomaron varias decisiones respecto a la formulación. Una de ellas fue denominarlas de forma breve y con un sustantivo - véase un ejemplo de la competencia Comunicación en tabla 2-: 
L. Lluch, E. Cano y L. Pacheco. Competencias Clave del Profesorado: diseño y validación del marco global competencial de los docentes

\section{Tabla 2}

Ejemplo de formulación de la competencia Comunicación.

\begin{tabular}{|l|l|l|}
\hline $\begin{array}{c}\text { Competencia } \\
\text { (dimensión) }\end{array}$ & \multicolumn{1}{|c|}{$\begin{array}{c}\text { Competencia } \\
\text { (descripción) }\end{array}$} & \multicolumn{1}{|c|}{ Descriptores o indicadores } \\
\hline 8. Comunicación & $\begin{array}{l}\text { Capacidad comunicativa con } \\
\text { los alumnos, con las familias, } \\
\text { con los colegas, con el equipo } \\
\text { directivo y con el resto de la } \\
\text { comunidad educativa. }\end{array}$ & $\begin{array}{l}\checkmark \text { Transmisión eficaz de información, ideas, } \\
\text { planteamiento de problemas y propuestas } \\
\text { de forma oral y escrita. } \\
\checkmark \text { Habilidad de relación y comunicación con } \\
\text { los estudiantes, familias, compañeros, } \\
\text { equipo directivo y el resto de la comunidad } \\
\text { educativa. } \\
\checkmark \ldots\end{array}$ \\
\hline
\end{tabular}

Nota. Fuente: Elaboración propia.

A la vez, los términos clave - competencia, estándar, indicador... - se definieron para establecer una comprensión compartida.

\subsection{Primera versión del marco competencial}

Las competencias se organizaron en dos tipos principales: específicas y transversales, siguiendo el currículum autonómico de Cataluña ${ }^{2}$ y otras propuestas internacionales -como, por ejemplo, Canadá ${ }^{3}$, Alemania ${ }^{4}$ y Australia ${ }^{5}-$

1. Las competencias clasificadas como específicas son características de la profesión docente. La competencia profesional es el conjunto de rasgos de personalidad, actitudes, conocimientos y habilidades que posibilitan el desempeño de actividades profesionales reconocibles en el mundo académico y en el mercado de trabajo (Álvarez, 2010). No hay competencia sin desempeño. Y, por ello, los elementos asociados a las competencias son: conocimientos (saber), actitudes (ser) y habilidades (hacer).

2. Una competencia interdisciplinaria se entiende como una competencia necesaria para el desarrollo comprensible del individuo y, además, proporciona el aprendizaje que es necesario en todos los campos profesionales. Por lo tanto, las competencias clasificadas como transversales son aquellas necesarias para cualquier campo profesional -autorreflexión y desarrollo profesional, tecnologías de la información y la comunicación, comunicación y compromiso ético-. No obstante, se ha decidido describirlos en relación con el contexto específico de los docentes de Educación Primaria.

\footnotetext{
${ }^{2}$ http://xtec.gencat.cat/ca/curriculum/primaria/curriculum/

${ }^{3}$ http://www.education.gouv.qc.ca/fileadmin/site_web/documents/dpse/formation_jeunes/54156_QEP_Chapitre3_LOW.pdf

${ }^{4}$ https://eacea.ec.europa.eu/national-policies/eurydice/content/teaching-and-learning-primary-education-18_en

${ }^{5}$ http://docs.acara.edu.au/resources/Developing_21st_century_competencies_Prof_Barry_McGaw.pdf
} 
L. Lluch, E. Cano y L. Pacheco. Competencias Clave del Profesorado: diseño y validación del marco global competencial de los docentes

\subsection{Validación por parte de expertos}

Un cuarto paso consistió en proceder a la validación por parte de dos expertos externos que fueron seleccionados en base a los criterios de experiencia y de accesibilidad. El perfil de los expertos participantes fue, por un lado, una mujer de Universidad Presencial, experta en decisiones basadas en evidencias y en evaluación y, por otro lado, una mujer de Universidad Online, experta en formación docente y en calidad.

Para la validación se creó un formulario ad hoc que preguntaba acerca de la importancia de cada competencia y de la alineación entre la etiqueta, la descripción o definición y los elementos descriptivos o indicadores (véase tabla 3):

Tabla 3

Formulario ad hoc para la validación de las competencias.

\begin{tabular}{|c|c|c|c|c|c|c|c|c|c|c|}
\hline \multirow[t]{2}{*}{ Competencias } & \multicolumn{2}{|c|}{$\begin{array}{l}\text { ¿Es esta una } \\
\text { competencia } \\
\text { clave para un } \\
\text { profesor? }\end{array}$} & \multicolumn{3}{|c|}{$\begin{array}{l}\text { ¿Cree que la } \\
\text { etiqueta es } \\
\text { apropiada } \\
\text { para la } \\
\text { competencia } \\
\text { descrita? }\end{array}$} & \multicolumn{2}{|c|}{$\begin{array}{l}\text { ¿Está de } \\
\text { acuerdo } \\
\text { con la } \\
\text { definición? }\end{array}$} & \multicolumn{2}{|c|}{$\begin{array}{l}\text { ¿Está de } \\
\text { acuerdo con } \\
\text { los elementos } \\
\text { descriptivos? }\end{array}$} & \multirow[t]{2}{*}{$\begin{array}{l}\text { Observaciones } \\
\text { y Comentarios }\end{array}$} \\
\hline & Sí & NO & Sí & & NO & Sí & NO & Sí & NO & \\
\hline \multicolumn{11}{|l|}{ Planning } \\
\hline \multicolumn{11}{|l|}{$\begin{array}{l}\text { Classroom } \\
\text { Management }\end{array}$} \\
\hline \multicolumn{11}{|l|}{ Assessment } \\
\hline \multicolumn{11}{|l|}{ Inclusion } \\
\hline \multicolumn{11}{|l|}{$\begin{array}{l}\text { Community } \\
\text { Action }\end{array}$} \\
\hline \multicolumn{11}{|l|}{ ICT } \\
\hline \multicolumn{11}{|l|}{ Communication } \\
\hline \multicolumn{11}{|l|}{$\begin{array}{l}\text { Professional } \\
\text { Development }\end{array}$} \\
\hline \multicolumn{11}{|l|}{$\begin{array}{l}\text { Ethical } \\
\text { Commitment }\end{array}$} \\
\hline & & & & Sí & NO & & OBSER & $\mathrm{AClO}$ & Y CON & ENTARIOS \\
\hline \multicolumn{11}{|c|}{$\begin{array}{l}\text { ¿Está de acuerdo con dividir las } \\
\text { competencias clave en competencias } \\
\text { específicas y transversales? }\end{array}$} \\
\hline \multicolumn{11}{|c|}{$\begin{array}{l}\text { ¿Está de acuerdo con la clasificación } \\
\text { actual de competencias específicas y } \\
\text { transversales? }\end{array}$} \\
\hline \multicolumn{11}{|c|}{$\begin{array}{l}\text { ¿Añadiría o eliminaría alguna } \\
\text { competencia? }\end{array}$} \\
\hline Comentarios ge & ales & & & & & & & & & \\
\hline
\end{tabular}

Nota. Fuente: Elaboración propia. 


\subsection{Segunda versión del marco competencial}

El marco fue ajustado siguiendo las sugerencias de los expertos, los cuales aportaban observaciones y comentarios mayoritariamente para especificar, ampliar y sustituir la información relativa a los elementos descriptivos de las competencias.

Esta segunda versión fue presentada en la reunión de lanzamiento del proyecto.

\subsection{Validación por parte del equipo internacional del proyecto}

En la reunión inicial se propuso acordar la lista de competencias clave de los maestros de primaria, así como su definición y elementos descriptivos. Con el propósito de llegar a una lista final consensuada de las competencias clave de los maestros de primaria, su definición y sus indicadores, en la sesión se procedió de la forma siguiente: a) se presentó el marco sugerido; b) se solicitó que, por equipos de cada país, se procediese a valorar la relevancia de cada una de las competencias propuestas en sus respectivos contextos nacionales así como su necesidad de disponer de una formación para su desarrollo; c) se solicitó también que, por equipos mixtos constituidos por miembros de diversos países, se comentase la definición de cada competencia y se ratificase/modificase/ampliase y d) se analizaron los resultados y se presentaron para la discusión grupal.

\subsection{Tercera versión del marco competencial}

Tras los ajustes, se llegó a una tercera y definitiva versión ${ }^{6}$ del marco competencial, que fue traducida al español y al chino.

\section{Conclusiones}

El Council of the European Union (2014) indica que la formación inicial debería proporcionar a los docentes las competencias clave que les permitan ser excelentes en su práctica, al tiempo que los motive a reforzarlas y actualizarlas a lo largo de su carrera profesional. En efecto, el Espacio Europeo de Educación Superior (EEES) opta por mejorar la calidad del proceso de enseñanza-aprendizaje incidiendo en aspectos como el profesorado, los programas, los estudiantes, las infraestructuras, la evaluación, entre otros; un nuevo paradigma que implica cambios en la formación del profesorado, dirigiendo la actividad docente hacia el desarrollo de capacidades, habilidades, actitudes y valores que posibiliten la formación competencial, donde adaptarse a los cambios sociales, económicos y tecnológicos, y transformar nuestra realidad. Consecuentemente, es preciso indagar, reflexionar y debatir, hacia dónde se deberían canalizar y orientar nuestras acciones, en la formación del profesorado, buscando un equilibrio entre calidad, capacitación y desarrollo profesional.

Uno de los retos de la implementación del enfoque basado en competencias es la necesidad de una coordinación entre las diferentes instituciones de formación, en concreto para una continuidad y

\footnotetext{
6 Véase la versión definitiva del marco competencial: http://tkcom.eu/gtkc-f/
} 
consistencia entre la secuencia de la formación docente inicial, temprana y de desarrollo. Esto es, a lo largo de los tres siguientes momentos: durante la formación profesionalizadora universitaria, en el momento de incorporarse al mundo laboral y como formación permanente a lo largo de su carrera docente.

Con el fin de optimizar el perfil competencial del alumnado universitario, es importante identificar el valor añadido que tiene un marco teórico global con un desglose de las competencias, según unos criterios, y en función de sus elementos descriptores. El hecho de tener al alcance un marco teórico global de las competencias clave del profesorado no solo es importante para identificar dichas competencias o capacitar a los docentes para desarrollarlas, sino también para que las instituciones se responsabilicen de su desarrollo competencial, certificación y validación a través de actividades de formación. Esto implica que los planes de estudios y los planes docentes adapten su diseño formativo bajo un enfoque basado en competencias; triangulando en todo momento la planificación, el diseño y la evaluación de la actividad docente, en coherencia con la metodología de enseñanza-aprendizaje, los objetivos y los contenidos de aprendizaje, las competencias clave, la evaluación, entre otros elementos.

Insistir, también, en la responsabilidad que recae en las facultades de educación y de formación del profesorado, como referentes en la creación de conocimiento - científico, pedagógico y didáctico-, para capacitar al alumnado universitario en el desarrollo de competencias clave y, en especial, para la transposición didáctica, puesto que la orientación y la calidad de esta formación profesionalizadora va a repercutir en todas las demás etapas educativas.

Es de suma importancia la validación por parte de expertos, de jefes académicos y de docentes universitarios para tener en cuenta la adaptación del marco teórico global en función del contexto nacional de cada institución de educación superior; para valorar su relevancia y su necesidad en la formación para su desarrollo. El hecho de llevar a cabo un proceso de validación permite acotar tanto la competencia como sus elementos descriptores para diseñarlo lo más preciso, práctico, objetivo, coherente y cohesionado posible. En definitiva, el detallado proceso de validación permite construir un sentido compartido de la competencia, atendiendo las diferencias y aproximaciones culturales.

En conclusión, esta contribución es una instancia a cómo diseñar un marco teórico global y su validación. La detallada descripción del proceso seguido permite su uso en futuras investigaciones o estudios.

\section{rganismos colaboradores}

Proyecto financiado por la Comisión Europea (Erasmus+).

\section{Referencias}

Álvarez, V. (2010). Evaluación de competencias en la Universidad [Documento no publicado]. Curso "Evaluación de competencias en el proceso enseñanza-aprendizaje". Secretariado de Formación y Apoyo a la Calidad. Universidad de Granada, junio-julio.

Black, P., y Wiliam, D. (1998). Inside the Black Box: Raising standards through classroom assessment. En R. Heilbronn y L. ForemanPeck (eds.), Philosophical perspectives on the future of teacher education (pp. 3-22). Wiley Blackwell. 
L. Lluch, E. Cano y L. Pacheco. Competencias Clave del Profesorado: diseño y validación del marco global competencial de los docentes

Bokdam, J., Van Den Ende, I., y Broek, S. (2014). Teaching Teachers: Primary Teacher Training in Europe State of affairs and outlook. European Parliament's Committee on Education and Culture. https://www.europarl.europa.eu/RegData/etudes/STUD/2014/529068/IPOL_STU(2014)529068_E N.pdf

Caena, F. (2011). Teachers' Core Competences: Requirements and Development. European Commission. https://ec.europa.eu/assets/eac/education/experts-groups/2011-2013/teacher/teachercompetences_en.pdf

Caena, F. (2014). Initial teacher education in Europe: an overview of policy issues. European Commission. https://ec.europa.eu/assets/eac/education/experts-groups/2014-2015/school/initial-teachereducation_en.pdf

Calvo-Bernardino, A., y Mingorance-Arnáiz, C. (2009). La estrategia de las universidades frente al Espacio Europeo de Educación Superior. Revista Complutense de Educación, 20(2), 319-394. https://doi.org/10.5209/rev_RCED.2013.v24.n1.41197

Conner, L., y Sliwka, A. (2014). Implications of Research on Effective Learning Environments for Initial Teacher Education. European Journal of Education, 49(2), 165-177. https://doi.org/10.1111/ejed.12081

Council of the European Union (2014). Conclusions of effective teacher education. EDUCATION, YOUTH, CULTURE and Council meeting, Bruselas. https://www.consilium.europa.eu/uedocs/cms_data/docs/pressdata/en/educ/142690.pdf

Darling-Hammond, L., y Bransford, J. (2005). Preparing teachers for a changing world: what students should learn and be able to do. Jossey-Bass.

Ding, S. (2016). Comparing the Key Teacher Competencies and the Training Process between China and Catalonia [Tesis doctoral, Universidad Autónoma de Barcelona]. https://dialnet.unirioja.es/servlet/tesis?codigo $=120826$

Hanushek, E. (2011). The Economic Value of Higher Teacher Quality. Economics of Education Review, 30(3), 466-479. https://doi.org/10.1016/j.econedurev.2010.12.006

Huang, Z. (2009). Thinking about Primary Teachers' Training Model. Teacher Education Research, 21, 7-12.

Marcelo, C. (2001). Aprender a enseñar para la Sociedad del Conocimiento. Revista Complutense de Educación, 12(2), 531-593.

OECD (2005). The definition and selection of key competencies. Executive Summary. https://www.oecd.org/pisa/35070367.pdf

OECD (2018). The Future of Education and Skills. Education 2030 [Web]. https://www.oecd.org/education/2030/

Olsen, J. P. (2005). The institutional dynamics of the (European) University. Arena centre for European Studies, University of Olso. 
L. Lluch, E. Cano y L. Pacheco. Competencias Clave del Profesorado: diseño y validación del marco global competencial de los docentes

Struyven, K., y De Meyst, M. (2010). Competence-based teacher education: Illusion or reality? An assessment of the implementation status in Flanders from teachers' and students' points of view. Teaching and Teacher Education, 26(8), 1495-1510. https://doi.org/10.1016/j.tate.2010.05.006

Park, J., y Tan, M. M. (coords.) (2016). Diverse approaches to developing and implementing competencybased ICT training for teachers: a case study. UNESCO Office Bangkok and Regional Bureau for Education in Asia and the Pacific https://unesdoc.unesco.org/ark:/48223/pf0000246003

Yang, T. (2009). Chinese Teacher Education Reform Strategic Position. Journal of the Chinese Society of Education, 6, 1-4.

Zhang, J. (2013). The research and development of Chinese teacher competences. Journal of Keilongjiang College of Education, 32, 35-36.

Zhou, D., Liji, W., Deng. S., y Xue, J. (2007). Reflections on the General Primary School Teacher Training. Teacher Education Study, 9, 55-59. 